\title{
Learning through Fictitious Play in a Game-theoretic Model of Natural Resource Consumption
}

\author{
Talha Manzoor, Elena Rovenskaya, Alexey Davydov, Abubakr Muhammad
}

\begin{abstract}
Understanding the emergence of sustainable behavior in dynamic models of resource consumption is essential for control of coupled human and natural systems. In this paper we analyze a mathematical model of resource exploitation recently reported by the authors. The model incorporates the cognitive decision-making process of consumers and has previously been studied in a game-theoretic context as a static two-player game. In this paper we extend the analysis by allowing the agents to adapt their psychological characteristics according to simple bestresponse learning dynamics. We show that, under the selected learning scheme, the Nash Equilibrium is reachable provided certain conditions on the psychological attributes of the consumers are fulfilled. Moreover, the Equilibrium solution obtained is found to be sustainable in the sense that no players exhibit free-riding behavior, a phenomenon which occurs in the original open-loop system. In the process, via a Lyapunov-function based approach, we also provide a proof for the asymptotic global stability of the original system which was previously known to be only locally stable.
\end{abstract}

Index Terms-Human-in-the-loop control, Game theory

\section{INTRODUCTION}

$\mathbf{S}$ YSTEMS such as complex Coupled Human And Natural Systems (CHANS) pose a notable challenge in being examined and ultimately controlled, owing to the fact that they span across multiple disciplines such as social sciences, engineering disciplines and the environmental sciences. The advent of technology and the realization of new control and sensing applications in such settings [1], [2] have especially accentuated the technical aspect of these systems. The CyberPhysical Social Systems (CPSS) [3], [2] concept provides an integrative language which not only covers the environmental and technical aspects, but also addresses the social aspect in CHANS. Such systems are now garnering the interest of not only the controls community but also other communities, albeit

This work was supported by the Center for Water Informatics \& Technology (WIT), Lahore University of Management Sciences (LUMS), Lahore, Pakistan and the Advanced Systems Analysis (ASA) Program of the International Institute for Applied Systems Analysis (IIASA), Laxenburg, Austria.

Talha Manzoor and Abubakr Muhammad are with the Department of Electrical Engineering and Center for Water Informatics \& Technology (WIT), Lahore University of Management Sciences (LUMS), Pakistan. $\{13060023$, abubakr\} @ lums.edu.pk

Elena Rovenskaya is with the Advanced Systems Analysis Program, International Institute for Applied Systems Analysis (IIASA), Laxenburg, Austria, and the Faculty of Computational Mathematics and Cybernetics, Lomonosov Moscow State University, Moscow, Russia. rovenska@iiasa.ac . at

Alexey Davydov is with the Department of Mathematics, National University of Science and Technology MISiS, Moscow, Russia and the Advanced Systems Analysis Program, International Institute for Applied Systems Analysis (IIASA), Laxenburg, Austria. davydov@vlsu.ru under different contexts and subtle differences in ideology [4], [5]. In this paper, we focus on the issue of sustainability of socio-ecological systems, a particular instance of CHANS. In particular we analyze the dynamics of a single renewable resource harvested by multiple consumers, recently proposed and studied by the authors in [6], [7].

Stankovic et. al. identify system identification as one of the primary challenges for the control of CPSS [4, Section 111]. Namely, they call for an extension of system identification techniques to adequately capture human behavior. The Human In The Loop (HITL) concept [5] addresses this issue by integrating human individuals as fundamental units of the system in contrast to the conventional approach of modeling human beings as exogenous inputs. In the same spirit, our selected model of resource consumption [6] employs research from social psychology [8], [9] to build a mathematical representation of consumer behavior. The social component of the model is dependent on psychological characteristics of the individual consumers such as their willingness to cooperate and the attribution of blame (nature or society) in the event of a resource crisis. The mathematical form of the model (see Equation (3), Section II-C) not only enables rigorous deductions and confirmation (or refusal) of theory, but also makes it amenable to analysis within multiple frameworks. For instance in the original study [6], the authors focused on the steady state properties of the model to analyze the emergence of undesirable phenomena such as free-riding. Also in [6], the model has been formulated as a static one-shot game in order to investigate the characteristics of sustainable communities under a predefined notion of sustainability. Subsequently, the authors have extended the model in [7] to explicify the underlying social network and also present various block models to simplify the analysis at various levels of abstraction.

Game theory offers a natural framework to study the strategic interactions between the consuming agents of our socioecological system. It not only complements the insights gained by viewing distributed systems (both natural and man-made) from a control theoretic perspective, but the outcomes predicted by game-theory's underlying paradigm of rational behavior can also serve as a benchmark for any control strategies that may be applied. While the Nash Equilibrium of the static one-shot game induced by the the selected model of natural resource consumption has already been examined in [6], the issue of how the agents actually reach the equilibrium has not been discussed prior to this study. This question is addressed by the theory of learning in games where the players change 
their strategies dynamically according to a particular learning scheme. A frequently studied family of learning dynamics involves players constructing a belief of each other's future strategies, based on the past strategies played, and playing a best response to the predicted strategy. This form of learning dynamics is commonly known as fictitious play and is perhaps one of the most extensively studied model of learning to date [10]. Here, after defining the game for the reformulated model, we extend the game-theoretic construction to a continuoustime repeated game and give a simple demonstration of how the agents can approach the Nash equilibrium by employing basic best response dynamics [11] (an elementary version of fictitious play). This is done by examining the stability of the basic system coupled with the learning adjustment process. We find that the resulting equilibrium is sustainable in the sense that the consumers do not exhibit any free-riding behavior (the notion of free-riding is adopted from previous studies and has been described herein). While the primary objective of this paper is to analyze the socio-ecological system coupled with the learning dynamics, in the process we also provide a proof for global stability of the original model, previously known to be only locally stable [6].

\section{The Coupled Socio-Ecological System}

Here we describe the system depicting the coupled resource and consumption dynamics. The basic model has originally been presented by us to the ecological modeling community [6], with a subsequent extension to explicitly incorporate the underlying social network [7].

\section{A. Ecological Sub-model}

The assumed setting is that of a single renewable resource whose stock dynamics are governed by the standard model of logistic growth [12]. The resource quantity at time $\tau$ is given by $R(\tau)>0$. In the absence of consumption, the stock grows at an intrinsic rate $\mathrm{r}>0$ and saturates at a carrying capacity $\mathrm{R}_{\max }$. The society consists of 2 consumer groups, where each group consumes the resource by exerting effort $e_{i}(\tau) \in \mathbb{R}$ where $i \in\{1,2\}$ (the full n-agent model can be found in [7]). The resource dynamics are given as follows

$$
\frac{d R}{d \tau}=\mathrm{r} R\left(1-\frac{R}{\mathrm{R}_{\max }}\right)-\left(e_{1}+e_{2}\right) R .
$$

It is important to note that the consumption efforts $e_{i}$ may take on both positive and negative values. While positive consumption corresponds to the extraction of the resource, negative consumption refers to any measure taken to replenish the resource base (detailed interpretations of negative effort and its effects on the resource stock are given in [6]).

\section{B. Social Sub-model}

In his ground-breaking theory of social comparison processes [9], Festinger posits that humans evaluate their decisions on the basis of both objective and social information. In the context of natural resource consumption, Mosler \& Brucks [8] interpret the objective information to be related to the state of the resource (the ecological factor) and the social information to be related to the consumption of other agents (the social factor). Similar to Mosler \& Bruck's computational model [8], we calculate the change in consumption as a weighted sum of the ecological and social factors. The relative weighing of these factors are modeled through certain psychological characteristics that portray the cognitive decision making process associated with resource usage. In what follows, we specify both factors and the psychological variables that determine the relative weighing of these factors to compute the change in consumption $\dot{e}_{i}$.

1) Ecological Factor: In their study [13], Rutte et. al. find that consumers harvest more when, according to their perception, the resource is in abundance, than when it is scarce. We model this effect by specifying the ecological factor as $R(\tau)-\mathrm{R}_{i}$ where $\mathrm{R}_{i} \in \mathbb{R}$ is the quantity of the resource below which $i$ considers it to be scarce and above which she considers it to be abundant. Note that $\mathrm{R}_{i}$ can lie outside the interval $\left[0, R_{\max }\right]$ which is justified by observing that $\mathrm{R}_{i}$ exists only in the mind of the consumer and does not represent a physical state of the resource. A negative value of $\mathrm{R}_{i}$ simply implies that $i$ considers the resource to always be in abundance no matter how low the actual stock is. Increasingly negative values amplify this effect via the factor $R(\tau)-\mathrm{R}_{i}$ (a similar argument holds for $\mathrm{R}_{i}>\mathrm{R}_{\max }$ ).

In the same study [13], the authors find that consumer behavior differs when they attribute resource scarcity to natureinduced reasons as compared to society-induced reasons. To elaborate, nature-induced reasons include natural disasters, adverse weather conditions, etc, whereas society-induced reasons include over-harvesting, pollution, wastage in consumption, and so on. We model the attribution of $i$ through $\mathrm{a}_{i}>0$ where $\mathrm{a}_{i} \rightarrow 0$ represents a group that attributes blame entirely to society, with increasing values of $a_{i}$ reflecting increasing attribution to nature. Rutte et. al. find that the consumers that attribute majority of the blame to nature, tend to give ecological information more importance and vice versa. We thus weigh the ecological factor for a single individual as $\mathrm{a}_{i}\left(R(\tau)-\mathrm{R}_{i}\right)$. In [7] we show that if the consuming agent is a group of consumers, then the ecological factor is weighed as $\mathrm{n}_{i} \mathrm{a}_{i}\left(R(\tau)-\mathrm{R}_{i}\right)$, where $\mathrm{n}_{i}$ is the size of the group and $\mathrm{a}_{i} \& \mathrm{R}_{i}$ represent the group's collective psychological characteristics.

2) Social Factor: Festinger [9] postulates that psychologically, humans find situations where others are very divergent from them, less appealing than situations where others are close to them (an effect that has subsequently been reiterated in other psychological studies as well [14]). For the two-agent case, we therefore define the social factor of $i$ to be given by the difference $e_{j}(\tau)-e_{i}(\tau)$ where $i, j \in\{1,2\}$ and $i \neq j$. The social factor is thus a measure of equality, with a lower level of equality (higher difference in consumption) prompting a higher change in consumption and vice versa.

The social-value orientation of group $i$ is represented by $\mathrm{s}_{i}>0$, where $\mathrm{s}_{i} \rightarrow 0$ represents an extremely non-cooperative consumer, with increasing values of $\mathrm{s}_{i}$ representing increasingly cooperative consumers. Social psychological studies [15] have indicated that cooperative individuals are concerned more with maximizing equality than non-cooperative ones, regard- 
less of their own outcomes. We thus weigh the social factor, as $\mathrm{s}_{i} \omega_{i j}\left(e_{j}(\tau)-e_{i}(\tau)\right.$, where $\omega_{i j} \in[0,1]$ represents the strength of the social tie directed from $j$ to $i$ with the constraint that $\sum_{j} \omega_{i j}=1$. If the consuming agent $i$ is an individual, then $\omega_{i i}=0$. However, as shown in [7], if the consuming agent $i$ is a group of consumers, then $\omega_{i i} \geq 0$ represents the strength of social bonding, or cohesion within the group, while $1-\omega_{i i}$ represents the group's bridging influence from the other groups.

Based on the above, the final dynamics of the consumption effort are given by

$\frac{d e_{i}}{d \tau}=\mathrm{n}_{i} \mathrm{a}_{i}\left(R-\mathrm{R}_{i}\right)+\mathrm{s}_{i} \omega_{i j}\left(e_{j}-e_{i}\right)$,

where $i \in\{1,2\}$ and $i \neq j$. It is interesting to note that while the dynamics have been derived from psychological first principles, they are similar to other models used in control and sociology such as quorum-sensing networks [16] and models of opinion formation [14].

\section{The Non-dimensionalized Socio-Ecological System}

We now reformulate (1) and (2) to express the coupled resource and consumption dynamics in terms of nondimensional states and parameters. Let $x(\tau)$ be the resource stock relative to $\mathrm{R}_{\max }$ and $y_{i}(\tau)$ be $i$ 's consumption relative to the growth rate $r$. The coupled socio-ecological system can then be represented as

$\dot{x}=(1-x) x-\left(y_{1}+y_{2}\right) x$,

$\dot{y}_{i}=\mathrm{b}_{i}\left(\left(1-v_{i}\right)\left(x-\rho_{i}\right)-v_{i}\left(y_{i}-y_{j}\right)\right)$,

where $i, j \in\{1,2\}, i \neq j, \mathrm{~b}_{i}=\left(\mathrm{n}_{i} \mathrm{a}_{i} \mathrm{R}_{\max }+\mathrm{rs}_{i} \omega_{i j}\right) / \mathrm{r}^{2}$, is called the sensitivity of $i$ and represents her openness to change in her consumption. $v_{i}=\mathrm{rs}_{i} /\left(\mathrm{n}_{i} \mathrm{a}_{i} \mathrm{R}_{\max }+\mathrm{rs}_{i} \omega_{i j}\right)$ is the social relevance of $i$ and represents the relevance $i$ places on social information relative to the relevance placed on ecological information, which is $1-v_{i}$ (note that $\left.v_{i} \in(0,1) \forall i\right) . \rho_{i}=\mathrm{R}_{i} / \mathrm{R}_{\max }$ is called the environmentalism of $i$ and $t=\tau / \mathrm{r}$ is the non-dimensionalized time. In what follows, we will work with this instance of the model given by (3).

\section{The Static Consumption Game}

Here we present the strategic confrontation between two consumer groups, previously defined in [6] as a static, oneshot game with two players. The game is defined as a 3-tuple $\mathcal{G}=\left\langle\mathcal{I}, \mathcal{S}_{i}, \pi_{i}\right\rangle$, where $\mathcal{I}=\{1,2\}$ denotes the set of players, $\mathcal{S}_{i}, i \in \mathcal{I}$ is the indexed strategy space for group $i$, and $\pi_{i}$ : $\mathcal{S}_{i} \times \mathcal{S}_{j} \rightarrow \mathbb{R}, i, j \in \mathcal{I}$ and $i \neq j$ is the indexed payoff function for $i$, defined on $\mathcal{S}_{i}$. In what follows, we define the elements of the game.

\section{A. Players}

The players of the consumption game are assumed to be two consuming agents $\mathcal{I}=\{1,2\}$. They may represent individual consumers or groups of consumers with homogeneous characteristics as outlined in [7]. The consumption dynamics for the two-agent society are given by (3).

\section{B. Payoffs}

The game-theoretic framework, similar to other theories of rational behavior, presumes that agents are able to construct a complete preference ordering over all possible outcomes. The ordering is mathematically expressed via an exogenous objective for each player, called the utility function or payoff. In many socio-ecological settings, it is common to assume that agents prefer outcomes where their consumption is more, to outcomes where they consume less [17]. In the past, many economists have taken sustainable development to imply infinite economic growth, which has been challenged by the concept of a steady state economy which, regardless of its attainability, may serve as an efficient tool to guide longterm policy making for real-world economies [12]. This gives special meaning to the steady state of (3). We thus define the payoff $\pi_{i}$ that each group receives, to be equal to the quantity of the resource that group harvests at steady state. Thus $\pi_{i}=\overline{\mathrm{x}} \overline{\mathrm{y}}_{i}$ where $\overline{\mathrm{x}}=\lim _{t \rightarrow \infty} x(t)$ and $\overline{\mathrm{y}}_{i}=\lim _{t \rightarrow \infty} y_{i}(t)$. In order to find the expressions for $\overline{\mathrm{x}}$ and $\overline{\mathrm{y}}_{i}$, we must determine any fixed points of (3) and investigate their stability.

1) Fixed point: It is found that system (3) has a unique fixed point which is given by

$$
\begin{aligned}
& \overline{\mathrm{x}}=\frac{\left(1-v_{1}\right) v_{2}}{v_{1}+v_{2}-2 v_{1} v_{2}} \rho_{1}+\frac{\left(1-v_{2}\right) v_{1}}{v_{1}+v_{2}-2 v_{1} v_{2}} \rho_{2}, \\
& \bar{y}_{i}=\frac{1}{2}-\frac{1-v_{i}}{2\left(v_{i}+v_{j}-2 v_{i} v_{j}\right)} \rho_{i}+\frac{\left(1-v_{j}\right)\left(1-2 v_{i}\right)}{2\left(v_{i}+v_{j}-2 v_{i} v_{j}\right)} \rho_{j},
\end{aligned}
$$

where $i, j \in\{1,2\}$ and $i \neq j$.

2) The Free-riding Phenomenon: The free-riding phenomenon [6] refers to situations where certain individuals (the self-reliants) sacrifice their consumption for the sustenance of the resource, while other individuals (the free-riders) continue to enjoy the benefits of consumption. Fixed-point (4) permits two possibilities. The "self-reliant" equilibrium consists of both groups harvesting at a positive rate $\left(\bar{y}_{1}>0\right.$ and $\bar{y}_{2}>0$ in (4)) and the "free-riding" equilibrium consists of one group harvesting at a positive and the other harvesting at a negative rate $\left(\overline{\mathrm{y}}_{i}>0\right.$ and $\overline{\mathrm{y}}_{j}<0$ for some $\left.i, j \in\{1,2\}\right)$. It can be seen that free-riding is excluded if the environmentalisms are equal i.e., $\rho_{1}=\rho_{2}$. If not, then free-riding is excluded only if the relatively environmental group has a social relevance $v_{i}$ greater than a certain threshold. We interpret this threshold as the reluctance of that group to subsidize the consumption of the other group which, consistent with social-psychological research, increases with an increase in cooperativeness of that group (see detailed calculations and interpretations in [6]).

3) Stability: Here we consider the stability properties of the fixed point (4) for system (3). We do this by transforming the system to an appropriate form and prove the validity of a Lyapunov function candidate. The proof is given by the following theorem.

Theorem 1: The system (3) is globally stable with all solutions tending to fixed point (4), if the following condition holds true

$\left(b_{1}-b_{2}\right)\left(b_{1} v_{1}-b_{2} v_{2}\right)+4 b_{1} v_{1} b_{2} v_{2}>0$. 
Proof: Let us define parameters $\mathrm{A}=\mathrm{b}_{1}\left(1-\mathrm{v}_{1}\right)+\mathrm{b}_{2}\left(1-\mathrm{v}_{2}\right)$, $a=b_{1}\left(1-v_{1}\right)-b_{2}\left(1-v_{2}\right), B=b_{1} v_{1}+b_{2} v_{2}, b=-b_{1} v_{1}+b_{2} v_{2}$, $\mathrm{D}=\mathrm{b}_{1}\left(1-v_{1}\right)\left(1-\rho_{1}\right)+\mathrm{b}_{2}\left(1-v_{2}\right)\left(1-\rho_{2}\right), d=-\mathrm{b}_{1}(1-$ $\left.v_{1}\right)\left(1-\rho_{1}\right)+b_{2}\left(1-v_{2}\right)\left(1-\rho_{2}\right), z_{0}=\ln \left(1-\frac{D B-b d}{A B+b a}\right), w_{0}=$ $\frac{1}{\mathrm{~B}}\left(-\mathrm{a} \frac{\mathrm{DB}-\mathrm{bd}}{\mathrm{AB}+\mathrm{ba}}-\mathrm{d}\right)$ and $\mathrm{u}_{0}=\frac{\mathrm{DB}-\mathrm{bd}}{\mathrm{AB}+\mathrm{ba}}+\frac{\mathrm{b}}{\mathrm{B}} \mathrm{w}_{0}$. It is easy to verify that $|\mathrm{a}|<\mathrm{A},|\mathrm{b}|<\mathrm{B}$, and $|\mathrm{d}|<\mathrm{D}$.

Defining new state variables $z=\ln x-\mathrm{z}_{0}, u=$ $\left(1+\frac{b}{B}\right) y_{1}+\left(1-\frac{b}{B}\right) y_{2}-\mathrm{u}_{0}$ and $w=y_{1}-y_{2}-\mathrm{w}_{0}$, system (3) can be transformed as follows

$\dot{z}=e^{z_{0}}\left(1-e^{z}\right)-u+\frac{\mathrm{b}}{\mathrm{B}} w, \dot{u}=\left(\mathrm{A}+\frac{\mathrm{ba}}{\mathrm{B}}\right) e^{z_{0}}\left(e^{z}-1\right)$,

$\dot{w}=\mathrm{a} e^{z_{0}}\left(e^{z}-1\right)-\mathrm{B} w$,

with the fixed point now at the origin. In order to prove the theorem we construct a Lyapunov function of the following form

$V=\left(e^{z}-z-1\right)+\mathrm{P} u^{2}+\mathrm{Q} w^{2}$,

which is positive for positive $\mathrm{P}, \mathrm{Q}$ everywhere except the origin. Thus if $\dot{V}$ is shown to be negative outside the origin for some positive $\mathrm{P}, \mathrm{Q}$, it would imply global stability of (6) and eventually of (3). Differentiating $V$ along the vector field of system (6) we get

$\dot{V}=e^{z} \dot{z}-\dot{z}+2 \mathrm{P} u \dot{u}+2 \mathrm{Q} w \dot{w}$.

Choose $\mathrm{P}=e^{-\mathrm{z}_{0}} /(2(\mathrm{~A}+\mathrm{ba} / \mathrm{B}))$. Due to the definition of $\mathrm{a}, \mathrm{A}, \mathrm{b}$ and $\mathrm{B}, \mathrm{P}>0$ so it does not violate the status of $V$ as a Lyapunov function candidate. Substituting this and (6) in the above equation gives us

$\dot{V}=-e^{\mathrm{z}_{0}}\left(e^{z}-1\right)^{2}+w\left(e^{z}-1\right)\left(\frac{\mathrm{b}}{\mathrm{B}}+2 \mathrm{Qaa} e^{\mathrm{z}_{0}}\right)-2 \mathrm{QB} w^{2}$.

Completing squares, we get

$$
\begin{aligned}
\dot{V}= & -\left(e^{\mathrm{z}_{0} / 2}\left(e^{z}-1\right)-\frac{w e^{-\mathrm{z}_{0} / 2}}{2}\left(\frac{\mathrm{b}}{\mathrm{B}}+2 \mathrm{Q} \mathrm{a} e^{\mathrm{z}_{0}}\right)\right)^{2} \\
& -\left(2 \mathrm{QB}-\frac{e^{-\mathrm{z}_{0}}}{4}\left(\frac{\mathrm{b}}{\mathrm{B}}+2 \mathrm{Qa} e^{\mathrm{z}_{0}}\right)^{2}\right) w^{2} .
\end{aligned}
$$

Now the condition $2 \mathrm{QB}-\frac{e^{-\mathrm{z}_{0}}}{4}\left(\frac{\mathrm{b}}{\mathrm{B}}+2 \mathrm{Qa}^{\mathrm{z}_{0}}\right)^{2}>0$ gives the derivative $\dot{V}<0$ outside the $u$-axis, and on this axis the vector field of (6) has value $(-u, 0,0)$, which is not tangent to the axis. Hence we get the desired global stability if this condition takes place, i.e., the above inequality is true for some positive Q, B. This condition can be expressed as

$\mathrm{p}\left(e^{\mathrm{z}_{0}} \mathrm{Q}\right):=4 \mathrm{a}^{2}\left(e^{\mathrm{z}_{0}} \mathrm{Q}\right)^{2}+\left(-8 \mathrm{~B}+4 \frac{\mathrm{ab}}{\mathrm{B}}\right)\left(e^{\mathrm{z}_{0}} \mathrm{Q}\right)+\frac{\mathrm{b}^{2}}{\mathrm{~B}^{2}}<0$.

Now, if $\mathrm{a}=0$, the inequality holds for any $\mathrm{B}>0$ by choosing $\mathrm{Q}$ to be sufficiently large. However if $\mathrm{a} \neq 0$ then due to $4 \mathrm{a}^{2}>0$ and $\mathrm{b}^{2} / \mathrm{B}^{2} \geq 0$, the inequality holds true for some positive $\mathrm{Q}, \mathrm{B}$ only if the vertex of the respective parabola belongs to the fourth quadrant, i.e.,

$-\frac{1}{8 \mathrm{a}^{2}}\left(-8 \mathrm{~B}+4 \frac{\mathrm{ab}}{\mathrm{B}}\right)>0$, and $-\frac{\Delta}{16 \mathrm{a}^{2}}<0$, where $\Delta$ is the discriminant of $\mathrm{p}\left(e^{\mathrm{z}_{0}} \mathrm{Q}\right)$. This translates to the following two conditions: 1) $-2 \mathrm{~B}^{2}+\mathrm{ab}<0$, and 2) $\mathrm{B}^{2}-\mathrm{ab}>0$. Substituting the values of $\mathrm{a}, \mathrm{b} \& \mathrm{~B}$, and realizing that $b_{1}, b_{2}, v_{1}$ and $v_{2}$ are all positive numbers, a sufficient condition for the condition $\mathrm{B}^{2}>$ ab (which enforces both 1 ) and 2) above) to hold is

$\left(b_{1}-b_{2}\right)\left(b_{1} v_{1}-b_{2} v_{2}\right)+4 b_{1} v_{1} b_{2} v_{2}>0$.

which completes the proof.

Theorem 1 thus shows that if condition (5) holds, then the payoff functions $\pi_{i}$ are well defined and equal to the limit of $i$ 's consumption as $t \rightarrow \infty$.

\section{Strategies}

It is assumed that each group is free to choose its level of environmentalism, represented by $\rho_{i} \in \mathbb{R}$. Thus the strategy set for each group $i$ is given as $\mathcal{S}_{i}=\left\{\rho_{i}\right\}$, which constitutes a game with continuous strategy space. Thus the payoffs $\pi_{i}$ are given by

$\pi_{i}\left(\rho_{i}, \rho_{j}\right)=\overline{\mathrm{x}}\left(\rho_{i}, \rho_{j}\right) \overline{\mathrm{y}}_{i}\left(\rho_{i}, \rho_{j}\right)$,

where $i, j \in\{1,2\}, i \neq j$ and $\overline{\mathrm{x}}, \overline{\mathrm{y}}_{i}$ are given by (4). Note that the strategy set does not include the variables $v_{i}$. The reason being that including $v_{i}$ as a second strategy variable along with $\rho_{i}$ results in the same Nash equilibrium as when $\rho_{i}$ is considered as the only strategy variable (see [6, Appendix A] for detailed calculations). Thus we treat $v_{i}$ as an exogenous parameter as this simplifies the analysis and does not effect the results.

\section{Best Response and Nash Equilibrium}

The best response of player $i$ is the strategy $\rho_{i}^{\#}$ that maximizes $\pi_{i}$ for a fixed strategy of the other player $j \neq i$. This is given as $\rho_{i}^{\#}=\operatorname{BR}_{i}\left(\rho_{j}\right)$ such that $\pi_{i}\left(\rho_{i}^{\#}, \rho_{j}\right)=$ $\max \pi_{i}\left(\rho_{i}, \rho_{j}\right)$. Solving this maximization problem yields the best response for $i$, which is given by

$\mathrm{BR}_{i}\left(\rho_{j}\right)=\frac{\left(v_{i}-v_{j}\right) \rho_{j}+\left(2 v_{i} v_{j}^{2}-v_{i} v_{j}-v_{j}^{2}\right)\left(1-\rho_{j}\right)}{2 v_{j}\left(v_{i}-1\right)}$.

The Nash equilibrium $\left(\rho_{1}^{*}, \rho_{2}^{*}\right)$ consists of all possible outcomes such that each strategy is a best response of the other. Thus this includes all such points $\left(\rho_{1}, \rho_{2}\right)$ where $\rho_{1}=\operatorname{BR}_{1}\left(\rho_{2}\right)$ and $\rho_{2}=\operatorname{BR}_{2}\left(\rho_{1}\right)$. Solving this condition algebraically gives us the following description for the Nash equilibrium.

$\rho_{i}^{*}=\frac{v_{i}\left(3 v_{j}-v_{i}-2 v_{i} v_{j}\right)}{\left(1-v_{i}\right)\left(v_{i}+v_{j}+2 v_{i} v_{j}\right)}$,

where $i, j \in\{1,2\}$ and $i \neq j$.

\section{Fictitious Play Learning in the Dynamic COnSUMPTiOn Game}

After previously defining the consumption game and deriving the Nash equilibrium we consider here how the players are able to achieve that equilibrium. We do this by allowing the players to adjust their strategies under fictitious play 
learning [10]. We employ fictitious play in its most basic form, commonly known as Best-response Dynamics, the Bestreply Process or Cournot Adjustment. While best-response learning is known to encounter some issues when applied as a model of learning in real-world scenarios [18] we select it here due to its relative simplicity and its ability to reveal certain dynamic aspects of system behavior that may recur when more sophisticated models of learning are applied. In particular, there exist various learning mechanisms for social beings that use prior distributions from past observations for the evolution of behavior [19], an effect that lies at the core of all fictitious play learning schemes in general.

\section{A. Learning Through Best-response Dynamics}

Here we observe the dynamics of the two-community system under best-response dynamics and discover that it indeed results in the actions converging to the Nash equilibrium in a distributed manner. Under these dynamics, the system propagates as follows.

$\dot{x}=(1-x) x-\left(y_{1}+y_{2}\right) x$,

$\dot{y}_{i}=\mathrm{b}_{i}\left(1-\mathrm{v}_{i}\right)\left(x-\rho_{i}\right)-\mathrm{b}_{i} v_{i}\left(y_{i}-y_{j}\right)$,

$\dot{\rho}_{i}=\mathrm{BR}_{i}\left(\rho_{j}\right)-\rho_{i}$,

where $i, j \in\{1,2\}, i \neq j$ and $\mathrm{BR}_{i}$ is given by (7). Under the dynamics given by $(9 \mathrm{~b})$, the groups' strategies $\rho_{i}$ are updated in direction of the best-response to each other's actions. Thus by definition, if an equilibrium $\left(\overline{\mathrm{x}}, \overline{\mathrm{y}}_{1}, \overline{\mathrm{y}}_{2}, \bar{\rho}_{1}, \bar{\rho}_{2}\right)$ of the above system exists it must be the Nash equilibrium, since at steady state $\bar{\rho}_{1}=\mathrm{BR}_{1}\left(\bar{\rho}_{2}\right)$ and $\bar{\rho}_{2}=\mathrm{BR}_{2}\left(\bar{\rho}_{1}\right)$. We find upon solving (9) for the equilibrium, that it is given by

$$
\begin{aligned}
& \overline{\mathrm{x}}=\frac{2 v_{1} v_{2}}{v_{1}+v_{2}+2 v_{1} v_{2}}, \bar{y}_{i}=\frac{v_{i}}{v_{i}+v_{j}+2 v_{i} v_{j}}, \\
& \bar{\rho}_{i}=\frac{v_{i}\left(3 v_{j}-v_{i}-2 v_{i} v_{j}\right)}{\left(1-v_{i}\right)\left(v_{i}+v_{j}+2 v_{i} v_{j}\right)},
\end{aligned}
$$

where $\bar{\rho}_{i}$ indeed constitutes the Nash equilibrium as given in (8). Note that in (10) both consumptions are positive i.e., $\overline{\mathrm{y}}_{1}>0, \overline{\mathrm{y}}_{2}>0$ which constitutes a self-reliant equilibrium as per the notion defined in [6]. This is in contrast to the equilibrium (4) of the open-loop system, which permits the qualitatively different steady state $\overline{\mathrm{y}}_{i}>0, \overline{\mathrm{y}}_{j}<0$ (the freeriding phenomenon [6]). Thus in a society following bestresponse dynamics, the consuming groups do not exhibit freeriding behavior as opposed to the open-loop system (3) where the strategies are specified as exogenous parameters.

\section{B. Convergence to the Nash Equilibrium}

In order to find whether the Nash equilibrium is reachable or not, we need to explore the stability properties of system (9). The global stability of (9a) has already been discussed above for constant $\rho_{i}$ 's. Since the subsystem (9b) does not depend on (9a), the stability of the overall system (9) can then be deduced by analyzing (9b) separately. More precisely, if $\rho_{1}$ and $\rho_{2}$ are guaranteed to reach a fixed point, then they may be treated as constants in (9a) which is known to be globally stable if inequality (5) holds. Thus, once (9b) reaches its steady-state, (9a) will also converge to its equilibrium (provided (5) holds) regardless of where the system was when that steady-state was achieved. Since (9b) is linear in $\rho_{1}$ and $\rho_{2}$, the stability can be deduced by analyzing the eigenvalues, which are given as

$\lambda_{1,2}=-1 \pm \sqrt{\frac{\left(v_{1}-v_{2}\right)^{2}+4 v_{1}^{2} v_{2}^{2}}{4 v_{1} \nu_{2}}}$.

It is obvious from (11), that both $\lambda_{1}$ and $\lambda_{2}$ are real. However, $\lambda_{1}$ may be positive which corresponds to unstable learning dynamics. It can be seen from Figure 1a that the learning dynamics are stable in a major area of the parameter space. Combining this information with the stability condition for (3), the overall criterion for stability of (9), in addition to (5), is given by

$\left(v_{1}-v_{2}\right)^{2}+4 v_{1}^{2} v_{2}^{2}-4 v_{1} v_{2}<0$.

This shows that the Nash Equilibrium is reachable via bestresponse dynamics, if (5) and (12) hold collectively.

\section{Simulation Results: Overcoming Free-riding Behavior}

Figures $1 \mathrm{~b}$ and 1c illustrate the learning process for two different points in the parameter space. Due to (10), when $v_{1}=v_{2}$ (Figure $1 \mathrm{~b}$ ) then the resource at steady state $\overline{\mathrm{x}}$ equals $\bar{\rho}_{1}=\bar{\rho}_{2}$. However when $v_{1} \neq v_{2}$ (Figure 1c), the resource $x$ follows the group with lower social relevance $v_{i}$. The case depicted in Figure 1c is particularly interesting and merits additional interpretation. As seen from the long-term behavior of the graphs, and from the mathematical form of the equilibrium (10), higher social-relevance $v_{i}$ implies a lower level of environmentalism $\rho_{i}$ and a higher steady state consumption $\overline{\mathrm{y}}_{i}$. This is in contrast to what the steady-state of the open-loop system (3) predicts i.e., cooperative individuals are usually associated with higher levels of environmental concern, and also consume less than non-cooperative individuals on average. Indeed this is what has been observed through different socialpsychological studies as well [6]. The closed-loop system (9) however predicts the behavior of the consumers if they act rationally according to the game defined in Section III. As mentioned in Section I, this highlights the relevance of game theory to distributed control problems, whereby the selected payoffs and learning scheme may be treated as design principles in the bigger problem of social control [11], [20] in order to obtain desired behavior. Indeed as we find here, the selected scheme eliminates the phenomenon of free-riding, which poses a significant challenge in the effective governance of natural resources [17].

\section{Discussion}

This paper uses the construct of a two-community society consuming a single natural resource, to formulate a continuous-time repeated game, in a cognitive model of consumer behavior. We demonstrate how the selected utility functions and learning scheme, converge to a steady state that eradicates free-riding behavior on part of the consumers. We also provide rigorous conditions on the psychological characteristics of the consumers that are required to reach this 


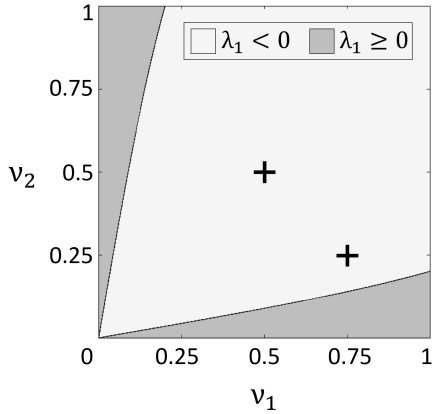

(a) The positivity of $\lambda_{1}$ from (11)
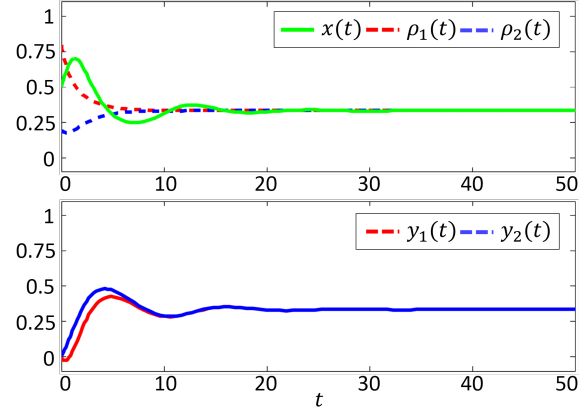

(b) Learning process for $v_{1}=v_{2}=0.5$.
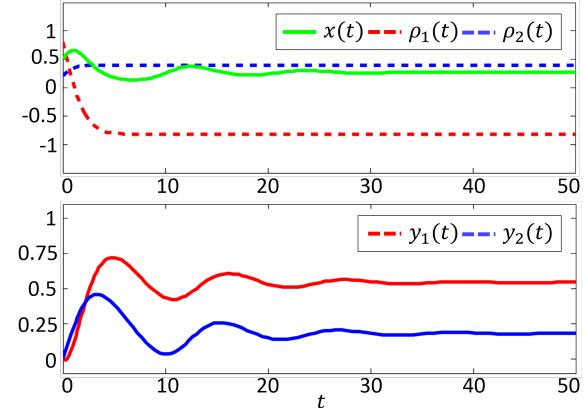

(c) Learning process for $v_{1}=0.75, v_{2}=0.25$.

Figure 1: left: The region of stability for $(9 \mathrm{~b})$. The cross-hairs correspond to points selected for the subsequent simulations. center: The learning process for identical $v_{i}$ 's. right: The learning process for different $v_{i}$ 's. In both simulations $b_{1}=b_{2}=1$, $x(0)=0.5, y_{1}(0)=y_{2}(0)=0, \rho_{1}(0)=0.8$ and $\rho_{2}(0)=0.2$. Note that since $\mathrm{b}_{1}=\mathrm{b}_{2},(5)$ holds true for all $v_{1} . v_{2}$.

steady state. These findings may potentially be used to inform policy making for natural resource management, especially since the study is based on one of the few fully justified mathematical models (at least to our knowledge) of socioecological couplings. We also hope that this study serves to instigate inquiry into other complex learning schemes that may enable convergence to equilibria with similar socially desirable features.

We now mention various shortcomings of the model which are important to note. Firstly, the assumptions made on information available to the consumers. In particular the basic model assumes that the consumers have perfect knowledge of the resource, each others' consumption and psychological characteristics. This is obviously a strict assumption and must be dealt with in future studies. Secondly, this paper has considered the time-variation of the environmental concerns of the consumers via a selected learning scheme. There also exist other psychological parameters in the model which are in a constant state of change in the real world. This offers another possible extension of the model. Finally, the current study considers a society with just two consumer groups. Whether or not the conclusions drawn here for the two-player game will also hold for an arbitrary number of players is definitely a compelling question for further investigation.

\section{ACKNOWLEDGMENT}

The authors are thankful to the anonymous reviewers for critically studying the initial version of the manuscript. Their constructive criticism and feedback greatly contributed to improving the final version of the paper.

\section{REFERENCES}

[1] A. Muhammad, "Managing river basins with thinking machines," in Norbert Wiener in the 21st Century (21CW), 2016 IEEE Conference on. IEEE, 2016, pp. 1-6.

[2] D. N. Crowley, E. Curry, and J. G. Breslin, "Closing the loop from citizen sensing to citizen actuation," in Digital Ecosystems and Technologies (DEST), 2013 7th IEEE International Conference on. IEEE, 2013, pp. 108-113.
[3] F.-Y. Wang, "The emergence of intelligent enterprises: From cps to cpss," IEEE Intelligent Systems, vol. 25, no. 4, pp. 85-88, 2010.

[4] J. A. Stankovic, "Research directions for the internet of things," IEEE Internet of Things Journal, vol. 1, no. 1, pp. 3-9, 2014.

[5] D. S. Nunes, P. Zhang, and J. S. Silva, "A survey on human-in-the-loop applications towards an internet of all," IEEE Communications Surveys \& Tutorials, vol. 17, no. 2, pp. 944-965, 2015.

[6] T. Manzoor, E. Rovenskaya, and A. Muhammad, "Game-theoretic insights into the role of environmentalism and social-ecological relevance: A cognitive model of resource consumption," Ecological Modelling, vol. 340, pp. 74-85, 2016.

[7] — "Structural effects and aggregation in a social-network model of natural resource consumption," Proceedings of the 20th IFAC World Congress, 2017.

[8] H.-J. Mosler and W. M. Brucks, "Integrating commons dilemma findings in a general dynamic model of cooperative behavior in resource crises," European Journal of Social Psychology, vol. 33, no. 1, pp. 119-133, 2003.

[9] L. Festinger, "A theory of social comparison processes," Human relations, vol. 7, no. 2, pp. 117-140, 1954.

[10] D. Fudenberg and D. K. Levine, The theory of learning in games. MIT press, 1998, vol. 2.

[11] J. R. Marden, J. S. Shamma et al., "Game theory and distributed control," Handbook of game theory, vol. 4, pp. 861-900, 2012.

[12] R. Perman, Natural resource and environmental economics. Pearson Education, 2003.

[13] C. G. Rutte, H. A. Wilke, and D. M. Messick, "Scarcity or abundance caused by people or the environment as determinants of behavior in the resource dilemma," Journal of Experimental Social Psychology, vol. 23, no. 3, pp. 208-216, 1987.

[14] N. E. Friedkin, A structural theory of social influence. Cambridge University Press, 2006, vol. 13.

[15] P. A. Van Lange, J. Joireman, C. D. Parks, and E. Van Dijk, "The psychology of social dilemmas: A review," Organizational Behavior and Human Decision Processes, vol. 120, no. 2, pp. 125-141, 2013.

[16] G. Russo and J. J. E. Slotine, "Global convergence of quorum-sensing networks," Physical Review E, vol. 82, no. 4, p. 041919, 2010.

[17] E. Ostrom, Governing the commons. Cambridge university press, 2015.

[18] T. Brenner, "Agent learning representation: advice on modelling economic learning," Handbook of computational economics, vol. 2, pp. 895-947, 2006.

[19] W. Hoppitt and K. N. Laland, Social learning: an introduction to mechanisms, methods, and models. Princeton University Press, 2013.

[20] T. Manzoor, S. Aseev, E. Rovenskaya, and A. Muhammad, "Optimal control for sustainable consumption of natural resources," IFAC Proceedings Volumes, vol. 47, no. 3, pp. 10725-10730, 2014. 